\title{
The use of anomalous X-ray diffraction as a tool for the analysis of compound semiconductors
}

\author{
Daniel M. Többens, Susan Schorr \\ Helmholtz-Zentrum Berlin für Materialien und Energie (HZB), Department Structure and Dynamic of \\ Energy Materials
}

\begin{abstract}
We provide a review about the current and previous use of anomalous diffraction of X-rays in the analysis of compound semiconductors. Among the large number of available techniques, those that have been used in successful experiments on this class of compounds are identified. An exhaustive overview of the compound semiconductor systems studied successfully is given and the kind of results derived in the experiments is discussed.
\end{abstract}

\section{Introduction}

The conductivity of semiconductors depends heavily on defects in their crystal lattice. In particular, the presence of atoms with a different number of electrons than those of the parent structure has very strong effect. Depending on whether the number of electrons is higher or lower, these impurity atoms act as either electron acceptors or donors. Semiconductors with donor impurities are called n-type, while those with acceptor impurities are known as $\mathrm{p}$-type. The $\mathrm{n}$ and $\mathrm{p}$ type designations indicate which charge carrier acts as the material's majority carrier. While donors provide extra, movable electrons, by the presence of an acceptor a vacant state (an electron "hole") is created, which can function as charge carrier. In pure one-element semiconductors, these impurities have to be specifically added to the crystal structure; this is called doping. In example, a silicon crystal doped with boron creates a p-type semiconductor whereas one doped with phosphorus results in an n-type compound.

In compound semiconductors, consisting of multiple chemical elements, the parent structure itself can provide both donors and acceptors. In example, copper zinc tin sulfide (CZTS) is a quaternary semiconducting compound of $\mathrm{I}_{2}-\mathrm{II}-\mathrm{IV}-\mathrm{VI}_{4}$ class. In this structure, $\mathrm{Cu}(\mathrm{I})$ and $\mathrm{Zn}(\mathrm{II})$, have different electric charges and can thus serve as $n$ - and $p$-type impurities for each other. As the two elements have very similar ionic radius, they can easily occupy their respective atomic sites in the structure. This kind of disorder naturally creates a huge density of defects. In addition, variations in the chemical composition can easily occur, resulting in off-stoichiometric compounds. In this case, site vacancies or interstitial atoms appear as a complicating factor. Controlling the distribution of the elements, and therefore the defects, in 
the crystal structure is a key requirement to the production of effective semiconductor compounds. The example is not untypical in that the elements in question are close together in the periodic system. The difference in the number of electrons is only one between copper and zinc, and the different charge makes the cations fully isoelectronic. As the X-ray scattering power of an atom is in first approximation proportional to its number of electrons, this makes it nigh-impossible to determine the distribution of the cations in the structure by conventional X-ray diffraction. In some cases, neutron diffraction can solve this problem (e.g. [1]), as neighboring elements can have very different neutron scattering factors [2]. This is the case for the Cu-Zn pair, but it not always an option (see table 1). Also, application of neutron diffraction is limited by the large amounts of sample typically required, which precludes its application to thin film samples. Further, long data collection times and the dwindling number of neutron sources worldwide limit this method to "corner stone" problems. Anomalous X-ray scattering offers an alternative.

\section{Basics of the method}

The core equation in the analysis of crystal structures by X-ray diffraction is the structure factor $\boldsymbol{F}(\boldsymbol{h})=$ $\sum_{n} f_{n} e^{i \varphi}$, which determines the intensity $I=|\boldsymbol{F}(\boldsymbol{h})|^{2}$ of a Bragg diffraction peak as a function of the $n$ atoms in the crystallographic unit cell. The phase $\varphi=2 \pi(h x+k y+l z)$ is determined by the Miller indices $h k l$ of the Bragg peak and the fractional coordinates $x y z$ of atom $n$. It should not be necessary to point out that the structure factor is a complex function to begin with and phase information is lost in the intensity of the Bragg peak. The interaction of the X-rays with the individual atoms compromising the crystal structure is subsumed in the atomic scattering factor $f_{n}$. This is the parameter central to all anomalous scattering methods.

The atomic scattering factor is generally expressed in the form $f=f_{0}+f^{\prime}+\mathrm{i} f^{\prime \prime}$. The first term, the atomic form factor $f_{0}$, describes classical elastic scattering, in which incident electromagnetic waves induce sympathetic vibrations in the electrons and the accelerated electrons emits radiation according to Maxwell's equations. The form factor $f_{0}$ is not dependent of the wavelength $\lambda$ of the radiation, but decreases with increasing scattering angle $\Theta$ as a function of $Q=4 \pi \sin (\theta) / \lambda$. This form factor is the Fourier transform of the electronic density in the atom. It tends toward the total number of electrons in the atom, thus the atomic number $Z$, in the forward scattering limit $(2 \Theta \rightarrow 0)$. For ions, corresponding changes have to be considered, and for heavy atoms a relativistic correction on the order of 0-2 e- must be subtracted, but this does not change the general picture. One of the foremost results of this is that atoms with similar number of electrons have similar scattering power. This makes them hard to distinguish in $\mathrm{X}$-ray diffraction experiments.

However, electrons in an atom are not completely free to respond to incident radiation; they can only occupy electronic orbitals with characteristic energies. As the energy of the incident photon approaches this energy, also its frequency approaches the atomic orbital frequency. The resulting resonance effects result in transitions of the electron from bound states to other accessible orbitals. This perturbs the atomic scattering. Such interactions also lead to photoelectric absorption whenever the x-ray energy exceeds the orbital binding energy, the absorption edge. This generates an ionized atom and a photoelectron. Subsequently, these photoelectrons recombine with the resulting ions, producing fluorescent $x$-rays with 
an energy characteristic of the electronic transition involved. The scattering perturbations from such orbital interactions add to the Thomson scattering with increments that have both amplitude and phase shift components and corresponding real and imaginary components, respectively $f^{\prime}$ and $f^{\prime \prime}$. These scattering perturbations are known as 'anomalous' scattering; however, they are normally present.

As anomalous scattering derives from core electrons, it is essentially independent of scattering angle, in contrast to normal scattering as expressed by the form factor. On the other hand, as anomalous scattering derives from resonance and to absorption, it depends strongly on $x$-ray energy and wavelength. Wavelength $\lambda$ and energy $E$ of a photon are related by the equation $E=h c / \lambda=12.3984(\mathrm{keV} / \AA) / \lambda$. In this paper we will use energy $E$, as this use is dominant in the literature. The resonance condition results in discontinuities of $f^{\prime}$ and $f^{\prime \prime}$ at the absorption edges (see figure1). It is these strong changes close to the absorption edges, which are used in the methods of anomalous diffraction. As the absorption energy is different for different chemical elements, this allows distinguishing between atoms with similar atom number. How the energy dependency of $f^{\prime}$ and $f^{\prime \prime}$ is utilized is the distinguishing feature between the various methods.

\section{Determination of anomalous scattering factors}

Common to all methods is the need to know the actual values of $f^{\prime}$ and $f^{\prime \prime}$ for any given energy. There is a wide range of ways to derive these values [3]; we will mention here only those used in actual experiments on compound semiconductors:

The spectra $f^{\prime}(E)$ and $f^{\prime \prime}(E)$ can be derived from quantum mechanical calculations. For isolated atoms, this has been done systematically for the complete periodic system. Widely in use are the theoretical approximations developed by Cromer and Liberman [4,5]. Values calculated on this basis are readily available from online databases (e.g. [6-8]) or are already tabulated in analysis software. Alternatively, software utilizing algorithms based on these approximations [9-11] can be used.

When experimentally tested, data from different sources were found to be of equal quality. In example, a joint refinement of the sulfosalt-like phase $\mathrm{Sn}_{4.11} \mathrm{Bi}_{22.60} \mathrm{Se}_{38}$ [12] from single crystal data collected at the Kedges of $\mathrm{Bi}, \mathrm{Sn}$, and off-edge compared the integrated database of JANA2006 [13] with anomalous dispersion correction terms from the NIST database [8], finding no significantly different results.

The approximations of Cromer and Liberman do not consider resonant transitions from core atomic orbitals to unoccupied orbitals. For atoms in a crystal structure, these outer orbitals are subject to changes due to the charge of ions and due to chemical bonding and coordination of the atom by its neighbors. While the theoretical values are quite accurate far away from the absorption edge, chemical bonding results in significant deviations close to the absorption edge. Depending on the particular crystal structure, these deviations can take the form of shifts of the absorption edge, broadening, and a fine-structure of peaks. In particular, the fine-structure is strong at energies above the absorption edge. Experimental techniques utilizing this energy range are affected stronger and thus have to expand more diligence in determining the correct values. Below the absorption edge, pre-peaks are typically weak and restricted to 
a few $\mathrm{eV}$ from the edge. For techniques utilizing only this range, often the tabulated values of Cromer and Liberman were found to be sufficient. Modern software for quantum mechanical calculations of the properties of crystals is capable of simulating the anomalous scattering spectrum and its fine structure. However, at this point this has not been used in published experiments. This is likely due to a still too high degree of specialized knowledge needed for the reliable conduction of these calculations.

It has been found that these values of the anomalous scattering factors are often sufficient for the kind of problems semiconductor analysis poses. A study regarding the treatment of $f^{\prime}[14]$ compared single crystal data taken at energies slightly below the respective K-edges of all elements compared for $\mathrm{CrSbSe}_{3}, \mathrm{PbTe}$, $\mathrm{SnSe}_{2}$, InSel, $\mathrm{SnTe}$, and $\mathrm{Sb}_{2} \mathrm{Te}_{3}$. It was found that tabulated or simple calculated correction factors differ from the real value by only one or two electrons. This is sufficient if the structural problem is less complex and the general trend is of more interest than the exact occupations.

For more demanding problems it is necessary to determine $f^{\prime}$ and $f^{\prime \prime}$ experimentally. In principle, any experiment making use of $f^{\prime}$ and $f^{\prime \prime}$ can also be used to determine these values. $f^{\prime}$ in example can be refined as a parameter in a crystal structure refinement, provided the site occupation factors for the respective chemical element are known [15]. A standard compound with structure similar to the actual sample, but with known relevant properties is typically used for this. In two papers on joint refinement of multiple diffraction data sets taken at different radiation energies $[16,17]$ the authors make the case, that the best method to treat the anomalous correction terms is the refinement of $f^{\prime}$ during the joint refinement of the data. They used dispersion correction factors $f^{\prime}$ and $f^{\prime}$ calculated with the program CROSSEC implemented in the CCP4 program suite and compared to values interpolated from various databases $[7,8]$. According to their results, "manually varying the values for $\Delta f$ in joint refinements shows that the refined element distribution is very robust and does not change more than a few standard deviations when $\Delta f$ is changed by about \pm 0.5 . Therefore, the overall result does not depend significantly on the exact values used. The best option was the refinement of $\Delta f^{\prime}$ for those values that are strongly affected by resonant scattering [...] and to keep all others as an average from different calculations". It can be assumed that this statement is only valid for high-quality data and sufficient over-determination, such as is the case in the respective experiments. In particular, it was found that using this method results in a 10-fold increase of estimated standard deviations for the atomic site occupancy factors due to strong correlation [14].

The most common experimental approach, however, utilizes X-ray absorption spectroscopy (XAS), that is, the experimental techniques known as XANES (X-ray absorption near edge structure) and EXAFS (Extended $\mathrm{X}$-ray absorption fine structure). For this, either the $\mathrm{X}$-ray absorption factor or the X-ray induced fluorescence spectrum is measured as a function of $\mathrm{X}$-ray energy around the respective absorption edge. Than the fundamental connection between scattering, absorption and fluorescence is utilized. According to Fresnel diffraction theory, the $f_{0}$ scattered wave is $\pi / 2$ out of phase with the incident wave and $f^{\prime \prime}$ is out by another $\pi / 2$; therefore, in the forward direction, the $f^{\prime \prime}$ component interferes destructively with the incident beam [18]. This is the physical basis for absorption. As a result $f^{\prime \prime}(\mathrm{E})$ is directly proportional to the absorption coefficient $\mu(\mathrm{E})$. Fluorescence, resulting from the re-emission of the absorbed energy, is also directly proportional to both $f^{\prime \prime}$ and the X-ray absorption coefficient $\mu$. Consequently, it is sufficient to determine either $f^{\prime}$ or $f^{\prime \prime}$ experimentally, as they can be derived from each other by Kramers-Kronig transformation [3]. Done properly, this approach delivers the highest quality of values for $f^{\prime}$ and $f^{\prime \prime}$ [14]. In 
particular, the necessary XAS measurements can be done using the actual sample. However, this approach is time-consuming and requires both specialized equipment and knowledge.

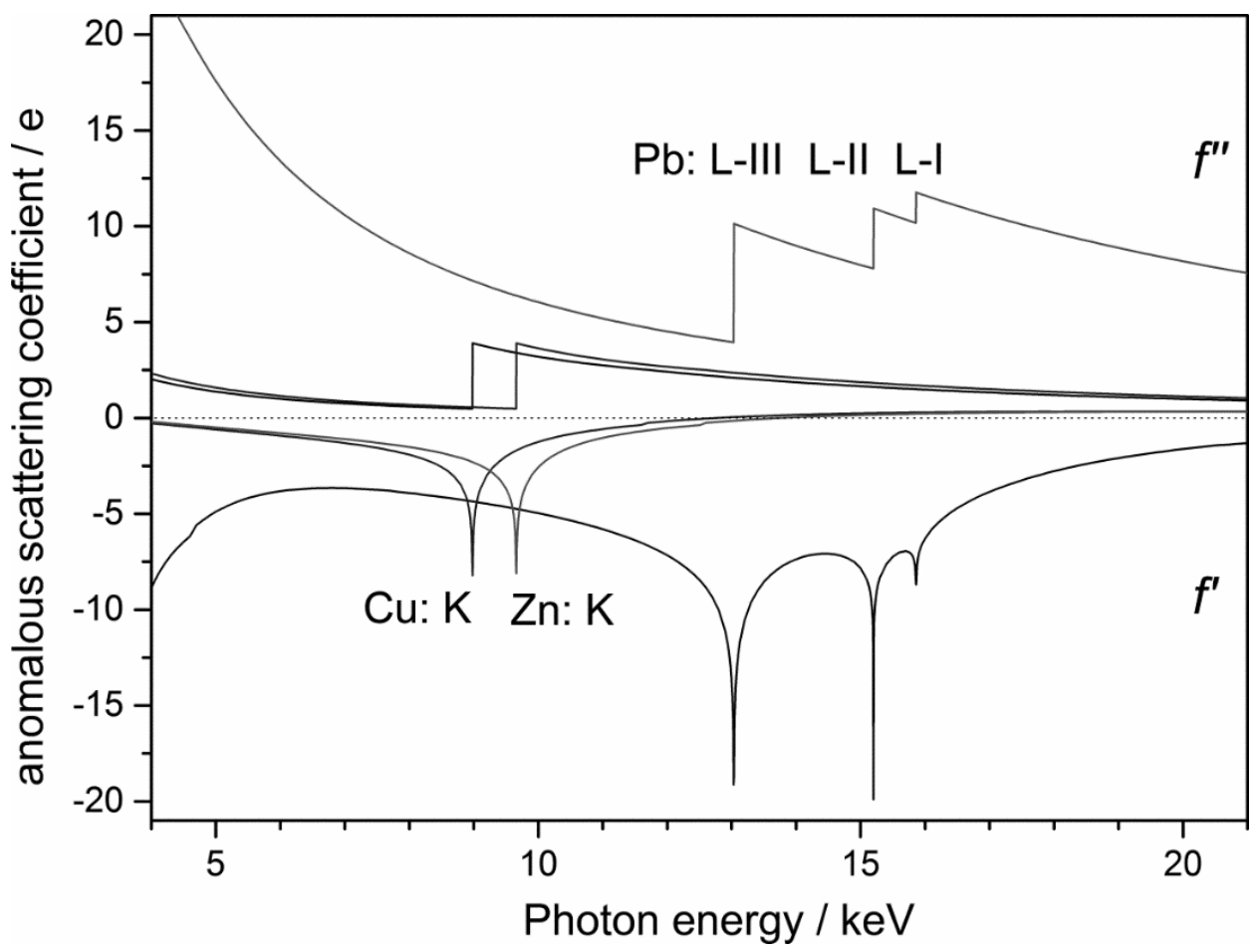

Figure 1. Exemplary energy dependency of anomalous scattering factors $f^{\prime}$ (bottom) and $f^{\prime \prime}$ (top) of copper, zinc and lead in the most commonly used energy range. K-absorption edges of $\mathrm{Cu}$ and $\mathrm{Zn}$ and L-edges of $\mathrm{Pb}$ fall into this range.

\section{Experimental methods}

Various analytical methods have been applied successfully to the study of compound semiconductors.

The experimental approach requiring the least adaption of the accustomed working style of crystallographers is probably the direct refinement of the crystal structure from diffraction data sets collected at energies close to the absorption edge. As anomalous diffraction is always present to some degree, it is already incorporated in the atomic scattering factor handling of common crystal structure refinement software, be it for single-crystal data or for Rietveld refinement of powder diffractograms. In addition, most software either has in-build tables of anomalous scattering factors or allows manual input of these values taken from external sources. Beyond this, no specialized knowledge is needed for this technique. Refinements can be done using a single dataset taken at only one selected energy. In this case anomalous scattering is used for contrast enhancement only and analysis of refined atomic occupation factors can be done in the same way as for non-isoelectronic atoms in normal X-ray diffraction. It is also possible to refine multiple datasets in a joint refinement. This allows the simultaneous refinement of multiple occupancies for any atomic site, limited only by the number of different chemical elements, for which a diffraction pattern at an absorption edge can be obtained (plus one data set collected off-edge). 
The use of multiple measurements at different energies at the same edge contains little additional information, as the relation between $f^{\prime}$ and total atomic scattering power $f=f_{0}+f^{\prime}+\mathrm{i} f^{\prime \prime}$ is monotone [15]. An exception would be measurements below and above the absorption edge, where $f^{\prime \prime}$ changes strongly. However, as discussed in the previous section, experiments of this kind are typically conducted below the absorption edge only, in order to avoid fine structure and fluorescence. Experiments generally confirm what is intuitive from the energy dependency (figure 1) of the anomalous scattering factors, that data collection should be done as close to the absorption edge as possible, where the amplitude of $f^{\prime}$ is highest [14]. This is limited by the energy resolution of the instrument and the onset of fine-structure pre-edge peaks. $f^{\prime}$, in contrast to $f_{0}$, does not decrease with increasing Q. Due to this, changes in $f^{\prime}$ have particular strong influence on the Bragg peaks at high diffraction angles. Data collection up to high diffraction angles and using short-wavelength radiation is thus desirable. In order to achieve the later, often one additional off-edge set is collected. Additionally, this also helps in lifting the correlation between site occupation factors and thermal displacements parameters of the atoms. The use of single-crystal over powder samples, if this is an option, is also desirable.

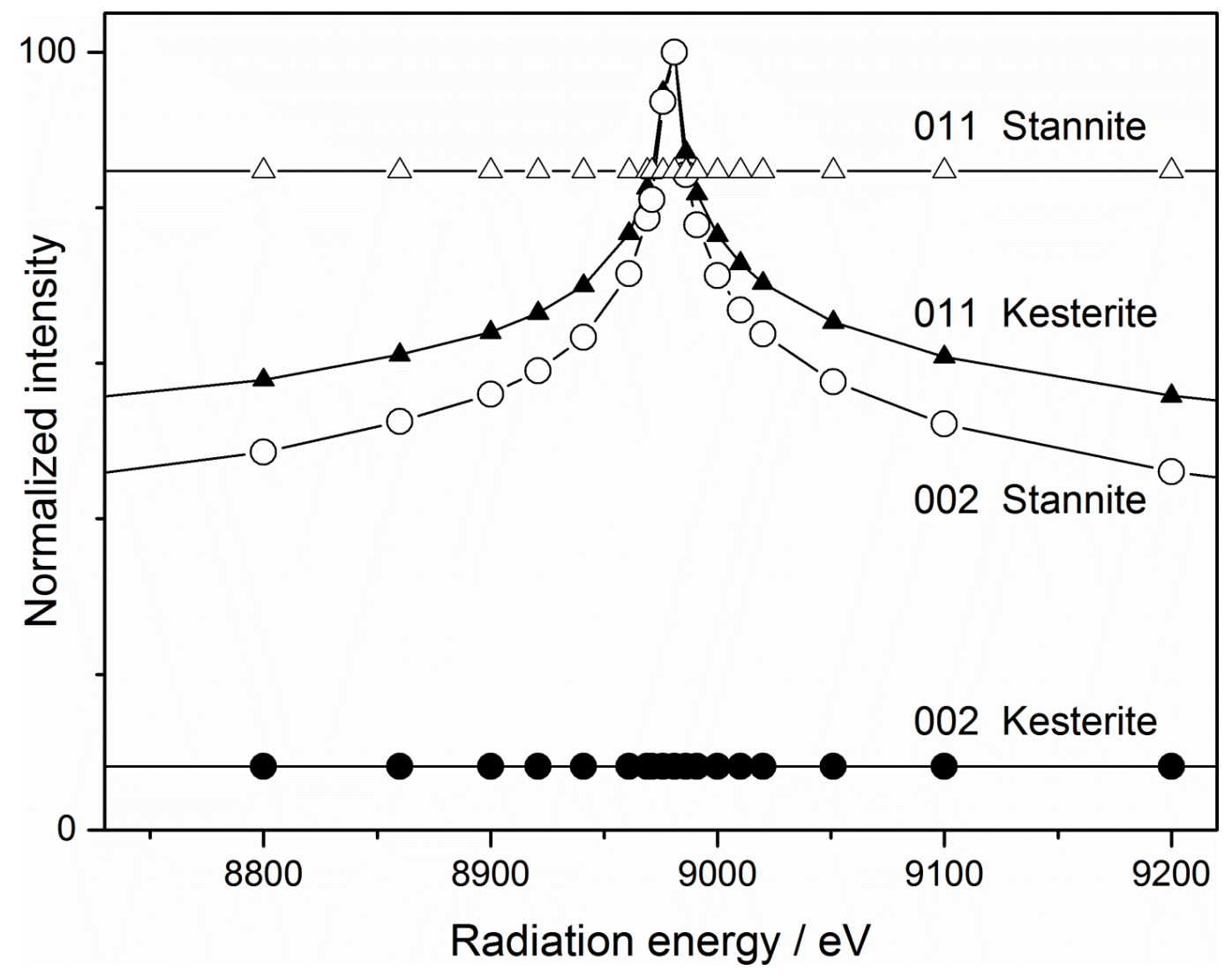

Figure 2: Calculated intensity variation at the $\mathrm{Cu}$ K-edge for the 002 and 011 Bragg peaks of $\mathrm{Cu}_{2} \mathrm{ZnSnS}_{4}$ in Kesterite (real) or Stannite (hypothetical) structure, normalized to the maximum intensity of the variable peak. 
The second frequently used technique is analyzing the energy dependence of the intensity of a single or small number of selected Bragg peaks over the absorption edge. For this the term Multiple-Edge Anomalous Diffraction (MEAD) has been coined [19]. The technique requires the collection of data at many energies, approaching a continuum (figure 2). In terms of experimental time, this is counterbalanced by the fact that it is not necessary to collect complete diffraction patterns. Data analysis is then done by fitting the structure factor of the model, given as a function of energy. In order to eliminate systematic errors from either the experiment or the model, it can be useful to analyze the intensity ratio of two selected peaks, from the same structure, a second phase or from a substrate, instead of the intensity itself. The advantage of this technique is that it allows very clearly to test and quantify particular features of the structure, provided a Bragg reflection can be found that is strongly dependent on this feature.

In terms of data collection, MEAD is basically undistinguishable from the widely used Diffraction Anomalous Near-Edge Structure spectroscopy (DANES) and its variant Diffraction Anomalous FineStructure Spectroscopy (DAFS), though the Fourier transformation approach inherent in these techniques strictly demands that the density of data points approaches continuum. Where MEAD aims at an analysis through diffraction theory, DANES and DAFS utilize analysis equivalent to XANES and EXAFS, respectively, in order to determine local structure details. Diffraction is used only to infuse site-specificity into the results. DANES and DAFS have been used both individually and in combination with other anomalous diffraction techniques on a number of semiconductors. A recent overview of the theoretical basics and the state of the art in these techniques can be found in "Site- and phase-selective $x$-ray absorption spectroscopy based on phase-retrieval calculation" [20].

Direct determination of the crystal structure, as widely used in the organic field [21] with methods like single-wavelength anomalous diffraction (SAD) and multiple-wavelength anomalous diffraction (MAD), is virtually absent in the field of compound semiconductors, where the basic crystal structure is typically known and the refinement of specific details is in the focus. The method has been adapted to the determination of the two-dimensional structures of very thin films of semiconductors from crystal trunctation rod (CTR) diffraction data [22].

Another specialized approach is the analysis not of the directly observed diffraction data, but of the intensity difference between Bragg reflections taken at the absorption edge and off-edge. This has been utilized in $\delta$-synthesis [23]. Without going into the details of the method, its practical use can be subsumed as akin to Difference Fourier diffraction analysis. In this widely used technique, the difference in the structure factor amplitude of the observed Bragg peaks from those calculated from the structure model is used to derive a scattering density map showing the remaining differences between the reals structure and the model. $\delta$-synthesis essentially does the same, leaving only the anomalous scattering density, which can then be analyzed. This specialized technique, too, has not seen wide usage.

A comprehensive overview over the theoretical background of the various methods can be found in Hodeaus et al. 2001 review paper "Resonant Diffraction" [18]. This paper also refers a wide range of experiments conducted prior to 2001, but with no particular emphasis on semiconductors. 


\section{Applications}

Printing a complete list of compound semiconductors and semiconductor alloy systems would be trite. Such lists can be readily found in handbooks [24], databases [25], and nowadays of course online lexica [26]; the later type of source should be considered a useful starting point only, as at least at this time they are still prone to contain glaring errors mixed into the useful information.

For a compound or alloy semiconductor material to be an interesting candidate for anomalous diffraction analysis, it has to contain at least two atomic species, which potentially can replace each other in the crystal structure. Exceptions to this are the rare cases in which MAD-derived methods have been used for the direct determination of the crystal structure.

Semi-conductor relevant groups of elements with very similar scattering power for normal X-rays are $\mathrm{Cu}$, $\mathrm{Zn}, \mathrm{Ga}, \mathrm{Ge}$ and $\mathrm{Ag}, \mathrm{Cd}, \mathrm{In}, \mathrm{Sn}$ and $\mathrm{Hg}, \mathrm{Tl}, \mathrm{Pb}, \mathrm{Bi}$. Within these, some elemental pairings have quite low contrast when neutron diffraction is applied (table 1). These include $\mathrm{Cu}-\mathrm{Ga}$ (6\% contrast), Ge-Cu (6\%), Sn$\mathrm{Ag}(5 \%), \mathrm{Tl}-\mathrm{Bi}(3 \%), \mathrm{Te}-\mathrm{Sb}(4 \%)$ and $\mathrm{Pb}-\mathrm{Tl}(7 \%)$. The contrast provided by anomalous $\mathrm{X}$-ray scattering can be higher.

\begin{tabular}{|c|c|c|c|}
\hline Element & Periodic number $\mathrm{Z}$ & $\begin{array}{l}\text { Bound coherent neutron } \\
\text { scattering length [2] } b_{c}\end{array}$ & $\begin{array}{l}\text { X-ray absorption edges used in } \\
\text { published work, and their } \\
\text { energies [6] }\end{array}$ \\
\hline $\mathrm{Mn}$ & 25 & $-3.73(2) f m$ & $\mathrm{~K}, 6.5390 \mathrm{keV}$ \\
\hline $\mathrm{Fe}$ & 26 & $9.54(6) \mathrm{fm}$ & K, $7.1120 \mathrm{keV}$ \\
\hline Co & 27 & $2.50(3) \mathrm{fm}$ & K, $7.7089 \mathrm{keV}$ \\
\hline $\mathrm{Ni}$ & 28 & $10.3(1) \mathrm{fm}$ & $\mathrm{K}, 8.3328 \mathrm{keV}$ \\
\hline $\mathrm{Cu}$ & 29 & $7.718(4) \mathrm{fm}$ & K, $8.9789 \mathrm{keV}$ \\
\hline $\mathrm{Zn}$ & 30 & $5.60(5) \mathrm{fm}$ & K, $9.6586 \mathrm{keV}$ \\
\hline Ga & 31 & $7.288(2) \mathrm{fm}$ & K, $10.3671 \mathrm{keV}$ \\
\hline $\mathrm{Ge}$ & 32 & $8.185(20) \mathrm{fm}$ & $\mathrm{K}, 11.1031 \mathrm{keV}$ \\
\hline $\mathrm{Ag}$ & 47 & $5.922(7) \mathrm{fm}$ & \\
\hline $\mathrm{Cd}$ & 48 & $4.87(5) \mathrm{fm}$ & \\
\hline In & 49 & $2.08(2) \mathrm{fm}$ & K, $27.9399 \mathrm{keV}$ \\
\hline Sn & 50 & $6.225(2) \mathrm{fm}$ & K, $29.2001 \mathrm{keV}$ \\
\hline $\mathrm{Sb}$ & 51 & $5.57(3) \mathrm{fm}$ & K, $30.4912 \mathrm{keV}$ \\
\hline Te & 52 & $5.80(3) \mathrm{fm}$ & $\mathrm{K}, 31.8138 \mathrm{keV}$ \\
\hline La & 58 & $8.24(4) \mathrm{fm}$ & L-I, $6.2663 \mathrm{keV}$ \\
\hline $\mathrm{Hg}$ & 80 & $12.692(15) \mathrm{fm}$ & \\
\hline $\mathrm{TI}$ & 81 & $8.776(5) \mathrm{fm}$ & L-III, $12.6575 \mathrm{keV}$ \\
\hline $\mathrm{Pb}$ & 82 & $9.405(3) \mathrm{fm}$ & L-III, $13.0352 \mathrm{keV}$ \\
\hline $\mathrm{Bi}$ & 83 & $8.532(2) \mathrm{fm}$ & \\
\hline
\end{tabular}

Table 1. Elements used in successful studies of compound semiconductors by anomalous diffraction and the absorption edges selected by the experimenters. Neutron scattering lengths are listed to provide a comparison with the most similar alternative method. 
The development of the use of anomalous scattering depends on the energy of the absorption edges [6] of the chemical elements under study on one hand and on the availability of X-ray radiation with this energy on the other. The energy of a given absorption edge increases with the atomic number of the element; e.g. the K-edge is at $2.472 \mathrm{keV}$ for sulphur, at $31.814 \mathrm{keV}$ for tellurium, and at $90.526 \mathrm{keV}$ for bismuth. The availability of the radiation with this energy is limited mostly by two effects: The maximum energy of the photons emitted as synchrotron radiation is limited by the energy of the charged particles circulating in the ring, with intensity dropping sharply already at lower energies. With modern light sources, however, it is nowadays possible to cover the whole range up to very high K-edges. At a given beamline, however, it might be necessary to use the L-absorption edges instead, which are at lower energies (e.g. Bi L-III $13.419 \mathrm{keV}$ ). The situation there is however complicated by the presence of three absorption edges, L-I, L-II, L-III, in close proximity. At the low energy end, limitations arise from increased absorption, in particular by air. While the transmission coefficient of air [7] at atmospheric pressure is 0.95 $\mathrm{cm}^{-1}$ for radiation with energy of $5 \mathrm{keV}$, it is only $0.53 \mathrm{~cm}^{-1}$ at $2 \mathrm{keV}$. Experiments using such low-energy radiation are thus only possible in vacuum or under helium. As absorption in materials used for X-ray windows, be it kapton, beryllium, or something else, increases similarly, this poses a considerable challenge to the experimentalist. As can be seen in table 1, experiments using anomalous X-ray diffraction to analyze compound semiconductors up to now have been restricted to the range of energies posing no such problems. This might change, as the use of ultrasoft X-ray radiation is advanced, in particular by the needs of the macrocrystalline community, which wants to use anomalous scattering at the K-edges of light elements like phosphor and sulphur in structure determination.

\section{Actual applications of the method}

\section{Early history}

The use of anomalous dispersion in X-ray diffraction analysis is connected with the study of semiconductors nearly from its very beginning. Not in the first known experiment using anomalous x-ray scattering, in which Mark and Szilard [27] demonstrated that diffraction from RbBr crystals differed selectively depending on the wavelength of irradiating $\mathrm{x}$-rays. $\mathrm{RbBr}$ crystallizes in the rock salt ( $\mathrm{NaCl})$ structure, in space group $F m \overline{3} m$. But in contrast to $\mathrm{NaCl}, \mathrm{Rb}^{+}$and $\mathrm{Br}^{-}$ions are isoelectronic and have very similar scattering power. Diffraction experiments using $\mathrm{X}$-rays from $\mathrm{Fe}, \mathrm{Co}, \mathrm{Cu}$ or $\mathrm{Zn}$ anodes thus failed to show the true unit cell resulting from the $\mathrm{Rb}-\mathrm{Br}$ order. This was different when $\mathrm{Sr}$ Ka radiation was used, whose energy falls between the $\mathrm{K}$ absorption edges of $\mathrm{Br}$ and $\mathrm{Rb}$. So while the first use of anomalous $\mathrm{x}$-ray scattering did not involve semiconductors, it already was applied to the most common problem it is applied to today in the field of compound semiconductors, the determination of site occupation factors of isoelectronic elements.

Three years later, Nishikawa and Matukawa demonstrated the hemihedry of Zincblende using anomalous diffraction [28]. Zincblende ( $\alpha$-ZnS, Sphalerite) is the cubic form of zinc sulfide; the metastable, hexagonal $\beta$-ZnS is called Wurtzite. Both sphalerite and wurtzite are intrinsic, wide-band gap II-VI semiconductor compounds. It was known already that Zincblende does not possess inversion symmetry, as crystals sometimes take a form without this symmetry element, e.g. the trigonal tristetrahedron [29]. In X-ray 
diffraction, this results in violations of Friedel's law only due to the presence of the anomalous component $f^{\prime \prime}$. Nowadays, of course, this effect is used routinely in normal single crystal structure determination to find the absolute conformation of the structure. However, with early photographic detectors and X-ray sources, the anomalous effect needed to be particularly strong to be observed. Nishikawa and Matukawa achieved this by selecting tungsten $L \beta_{1,2}$-radiation, which at $9.672 \mathrm{keV}$ and $9.962 \mathrm{keV}$ [30] is just a few eV

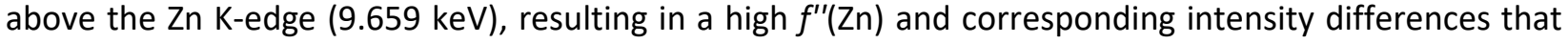
were not observed for the peaks produced by $\mathrm{W} \mathrm{K}-\alpha$ radiation. Unfortunately for the authors, this publication gathered little attention.

It is thus the 1930 experiment of Coster, Knol and Prins [31] on the same compound, which is commonly referred to as the first experiment utilizing anomalous dispersion [18, 32]. In this otherwise very similar experiment, a gold tube was used; the K-edge of zinc lies between $A u L \alpha_{2}(9.628 \mathrm{keV})$ and $A u L \alpha_{1}(9.713$ $\mathrm{keV})$. However, while the use of the imaginary component $f^{\prime \prime}$ of the scattering factor became widespread in the field of organic crystallography in the form of methods of structure determination by singlewavelength anomalous diffraction (SAD) and multiple-wavelength anomalous diffraction (MAD) [21], it has remained a rarity in the field of compound semiconductors. Here the use of changes in $f^{\prime}$ is dominant.

As far as we could find, modern application of anomalous dispersion to compound semiconductors started in 1984 with an attempt to determine the $\mathrm{Pb} / \mathrm{Bi}$ distribution in the crystal structure of $\mathrm{Pb}_{3} \mathrm{Bi}_{2} \mathrm{~S}_{6}$ (Lillianite) [33]. The experiment was conducted at the Photon Factory in Japan, utilizing radiation with energy close to the $\mathrm{Pb}$ L-III absorption edge. However, the experiment was finally unsuccessful, as experimental conditions, in particular the energy resolution available, at that time were not sufficient.

Other early experiments also fall in the task category of identification of the atom type occupying a crystallographic site. As the sought results are more qualitative than quantitative, the necessary data quality is not particularly high.

In the course of the development of $\delta$-synthesis, the method was applied to the then already known crystal structure of galenobismutite $\left(\mathrm{PbBi}_{2} \mathrm{~S}_{4}\right)$ [23]. The possibility to distinguish between lead $(\mathrm{Z}=82)$ and bismuth $(Z=83)$ was demonstrated as proof of concept. Galenobismutite is currently investigated as a promising thermoelectric compound [34].

The first successful application to an real, previously unknown problem was only conducted in 1994 [35]. In there, $\delta$-synthesis is applied to the determination of the distribution of copper and nickel in the mineral Mückeite $\left(\mathrm{CuNiBiS}_{3}\right)$, a ternary sulfosalt. Single crystal data collected at the HASYLAB Five-CircleDiffractometer [36] using energies of $8.316 \mathrm{keV}$ and $8.041 \mathrm{keV}$, close to and farer below the Ni-K-edge, were used. However, $\delta$-synthesis with is specialized approach to data analysis did not receive wider acceptance in the field of semiconductors.

\section{Element disorder and vacancies}

Most recent experiments attempt refinement of the site occupation factor. Even if determination of the chemical species occupying a site is the primary goal and antisite disorder and vacancies are not expected, 
conclusively demonstrating their absence is desirable. In other cases, determination of the density of point defects is the explicit purpose of the anomalous diffraction experiment. This is especially relevant for semiconductors, as those defects act as donor or acceptor impurities. A defining requirement for these experiments is the need to achieve very low uncertainties in order to reach a satisfying relative uncertainty of a small density of antisite atoms or vacancies.

The only other study on sulfosalts employing anomalous X-ray scattering employed joint refinement of multiple datasets in an analysis of cation disorder and vacancies in the sulfosalt-like phase $\mathrm{Sn}_{4.11} \mathrm{Bi}_{22.60} \mathrm{Se}_{38}$ [12]. Single crystal data were collected at beamline ID11 of the ESRF (Grenoble) using very hard X-rays. Due to this, the K-edges of the respective elements could be used instead of the L-edges: $29.203 \mathrm{keV}$ (Sn$\mathrm{K}$ edge), $90.456 \mathrm{keV}$ (Bi-K edge), and $81.659 \mathrm{keV}$ (off-edge). A joint refinement of the three synchrotron datasets and an additional laboratory dataset measured with $\mathrm{Ag}-\mathrm{K} \alpha$ radiation) was conducted with JANA2006 [13]. This experimental setup allowed the successful refinement of Bi and Sn occupation of six symmetry-independent atomic sites to uncertainties, which not only described the partly ordered distribution of the cations, but also revealed the presence of about $5 \%$ vacancies on cation positions.

The near complete absence of antisite disorder and vacancies in $\mathrm{SnSb}_{2} \mathrm{Te}_{4}$ [16] was shown by resonant single-crystal diffraction using synchrotron radiation with wavelengths near the K-absorption edges of the elements present and additional non-resonant data (both synchrotron and laboratory Mo-K $\alpha$ ). Refinement of site occupancies for all atoms on all sites was done with a joint refinement of all five datasets.

Distribution of the elements within the layered structure of $\mathrm{Ge}_{3} \mathrm{SblnTe}_{6}$ has been analyzed by single-crystal $\mathrm{X}$-ray diffraction using anomalous scattering at $\mathrm{K}$ edges of $\mathrm{Sb}$ and Te [37]. Single-crystal X-ray diffraction using synchrotron radiation at the $\mathrm{K}$ edges of $\mathrm{Sb}$ and $\mathrm{Te}$ (exploiting anomalous dispersion) yields precise information on the element distribution in the trigonal layered structure of Ge3SbInTe6 (R3m, $Z=3$, $a=4.19789(4) \AA, c=62.1620(11) \AA$ ). The structure is characterized by van der Waals gaps between distorted rocksalt-type slabs of alternating cation and anion layers. The cation concentration is commensurately modulated

\section{Solid solutions}

Refinement of the atom distribution in solid solutions is a subtopic of chemical disorder refinement. Particular to this task is that generally the chemical composition of the sample is well known and vacancies can be excluded. This reduces the number of independent occupation factors for any given atomic side, thus simplifying the analysis.

Solid solutions of silver indium telluride semiconductors with cadmium indium telluride have been analyzed by joint Rietveld refinement making use of anomalous scattering of all four elements involved $[38,39]$. The K-edges of these species lie in the range of $25-32 \mathrm{keV}$. This use of hard X-ray radiation is beneficial for the overall quality of the refined data, as the accessible Q-range is wider. This is of particular relevance for the powder diffraction analysis used in this case, where the method limits the quality of the results. In this study only one energy each slightly below the edges was used for diffraction. Together with 
a measurement away from the edges, up to five data sets per sample were collected (four for pure end members of the series): $35.004 \mathrm{keV}$ (away from edges), $31.822 \mathrm{keV} \mathrm{(<} \mathrm{Te-K),} 27.946 \mathrm{keV}(<\mathrm{In}-\mathrm{K}), 26.715$ $\mathrm{keV}(<\mathrm{Cd}-\mathrm{K}), 25.522 \mathrm{keV}(<\mathrm{Ag}-\mathrm{K})$. Note that the off- edge data were collected at an energy above the absorption edges. This results in fluorescence, leading to an increased background. However, this is unavoidable in any case in the general, as e.g. the data collection at the $\mathrm{Cd}-\mathrm{K}$ edge necessarily takes place at an energy causing fluorescence from the silver in the sample. The same holds for most other energies; the one data set without fluorescence in this experiment is the one take at the lowest energy, at the Ag-K edge. However, the beamline used, ID 31 (ESRF, Grenoble, France) [40] employs an array of point detectors, each one equipped with a $\mathrm{Si}(111)$ analyzer crystals. This removes the increased background from fluorescence scattering. Anomalous dispersion correction terms in this experiment were calculated from the characteristic $\mathrm{X}$-ray fluorescence of the respective element as a function of the wavelength of the incident radiation, employing the Kramers-Kronig transformation provided by the program $\mathrm{CHOOCH}$ [10]. It is unknown whether this had significant beneficial influence on the results.

Compounds in the Ge-Sb-Te-system, called GST materials, with the general formula (GeTe) ${ }_{x} \mathrm{Sb}_{2} \mathrm{Te}_{3}$ are widely used as phase-change materials (PCM) in opto-electronics. Sn-substituted GST materials have been investigated, because their performance can be enhanced by substituting Sn into the structure [41, 42].

In an experiment very similar to the one described above, the solid solution series between $\mathrm{GeSb}_{2} \mathrm{Te}_{4}$ and $\mathrm{SnSb}_{2} \mathrm{Te}_{4}$ [43] was analyzed. High-resolution synchrotron data measured at the K-absorption edges of Sn, $\mathrm{Sb}$ and Te combined with data measured at off-edge wavelengths were jointly refined. The distribution of $\mathrm{Sb}$ and Ge over the available sites could be established from this and the absence of significant antisite disorder could be verified for the analyzed samples.

Germanium and tin doped layered antimony tellurides [17] have also been analyzed, using joint refinement of single crystal data collected at the K-edges of all three elements and off edge in the case of Sn-doping. For Ge-doping, only off edge data and data at the Sb K-edge were collected. These layered structures of the type $39 \mathrm{R}-(\mathrm{Sn}, \mathrm{Ge})_{0.067} \mathrm{Sb}_{0.667} \mathrm{Te}_{0.266}$ have superstructures with very large unit cells $(c \approx 75$ $\AA)$; the use of very high quality data is thus mandatory for any structure refinement.

Joint refinement experiments on $\mathrm{Ge}_{1-x} \mathrm{Sn}_{x} \mathrm{Sb}_{2} \mathrm{Te}_{4}$ single crystals [44] used only three energies, $22.000 \mathrm{keV}$ (off edge), $30.477 \mathrm{keV}$ (< Sb K), and 31.808 (< Te K) skipping data collection at the $\mathrm{Sn}$ - and Ge-edges. This is sufficient, as the distinction of $\mathrm{Sn}, \mathrm{Sb}$ and Te is possible by changing the scattering power of only two of the three elements. Germanium is a much lighter element and has significantly lower scattering power to begin with. In the same paper, the temperature dependency of the disorder was analyzed. As the instrumentation did not allow for a realignment of the sample after a change in energy, only data at the $\mathrm{Sb}$-edge were collected. This is sufficient, as the overall sample composition does not change and can thus be fixed.

\section{Order-disorder phase transitions}

Besides the tellurides, the other class of compound semiconductors analyzed heavily by anomalous scattering is copper zinc tin sulfides (CZTS) and selenides (CZTSe). CZTSSe has received increased attention 
in recent years as promising alternative for absorber layers in thin film solar cells $[45,46]$. By varying the fractions of copper, zinc, and tin, vacancies and anti-sites can be induced. Most experiments successfully attempting direct quantification of cation distribution up to now has been done from neutron powder diffraction [47-50]. The neutron scattering lengths of copper and zinc are sufficiently different from each other, allowing distinction of the two species. Among other things it was settled in these papers that both CZTS and CZTSe compounds crystallize in the kesterite structure instead of in the alternatively proposed stannite structure[47]. Both structure candidates are derived from the sphalerite type by cation ordering. While the occupation of the heavy tin is readily determined by normal X-ray diffraction, $\mathrm{Zn}(\mathrm{II})$ and $\mathrm{Cu}(\mathrm{I})$ are hard to distinguish.

The first attempt to apply anomalous diffraction to was performed at BL19B2 in SPring-8 [51]. They analyzed thin film samples of CZTS turned into powder diffraction by scrapping of the film. Obviously it would have been hard to obtain the amount of material needed for neutron powder diffraction. Instead the X-ray energy dependency of the intensity of a few selected Bragg peaks was measured in the energy range $8.270-8.920 \mathrm{keV}$, energies slightly lower than the $\mathrm{Cu}$ K-edge. The observed change in intensity was then compared with simulations based on the Kesterite and Stannite structures. The two structure types differ only in the distribution of $\mathrm{Cu}$ and $\mathrm{Zn}$, with Sn-Zn- and Cu-layers in Stannite and Sn-Cu- and Cu-Znlayers in Kesterite. This experiment can be subsumed as hettotype determination. It is a common phenomenon that the structure of a compound is a derivative structure (hettotype) of a basic structure (aristotype) with fewer elements. The aristotype in this case is the cubic Sphalerite (ZnS) structure, which is the aristotype to the chalcopyrite $\left(\mathrm{CuFeS}_{2}\right)$ structure, which in turn has as hettotypes the stannite $\left(\mathrm{Cu}_{2} \mathrm{FeSnS}_{4}\right)$ and the kesterite $\left(\mathrm{Cu}_{2} \mathrm{ZnSnS}_{4}\right)$ structures. It could be confirmed that this sample, too, had Kesterite structure distribution. Note that the analysis method applied in this work technically could be classified as MEAD, albeit with a rather small number (7) of data points, and data collection only at one, the low energy side of the absorption edge.

In a follow-up paper [52] the samples were analyzed by Rietveld refinement of complete powder diffraction patterns collected at $8.920 \mathrm{keV}$. This is the energy of the previous work, which is most close to the absorption edge. This maximizing of the amplitude of $f^{\prime}$ is in agreement with recommendations in the literature [14]. Note that despite its earlier publication date, this is actually a follow up paper citing the previous one. With this, the contrast between $\mathrm{Cu}$ and $\mathrm{Zn}$ is sufficiently increased to refine their relative site occupation factors. However, with data taken at a single energy, additional restrains need to be induced; it is not possible to refine the occupation of any given site by $\mathrm{Cu}, \mathrm{Zn}$, and vacancies simultaneously without at least two different data sets. In this particular case the total occupation of the sites was assumed. With this method they could demonstrate for the different compositions of the samples changing degrees of $\mathrm{Cu} / \mathrm{Zn}$ ordering between complete order and complete disorder as well as the preference of additional Cu for the Sn site, of additional Zn for the Cu-site within the Sn-Cu-layers.

When it was reported that physical properties were linked to a low-temperature phase transition (Scragg et al., 2014), potentially offering a chance of control by synthesis conditions [53], and this sparked interest in the nature of this phase change. As cation ordering within the $\mathrm{Cu}-\mathrm{Zn}$ plane was a likely candidate, besides neutrons [50], anomalous diffraction was also applied to the problem. In a single crystal study on CZTS [54], data were collected at radiation energies of $8.968 \mathrm{keV} \mathrm{(<Cu} \mathrm{K)} \mathrm{and} 9.653 \mathrm{keV}$ (<Zn K). Off-edge 
data were collected at $18.5 \mathrm{keV}$, allowing the collection of data up to high $\mathrm{Q}$. A joint refinement found complete disorder within the $\mathrm{Cu}-\mathrm{Zn}$ plane. This is in agreement with later results $[50,55]$, as the sample the single crystal was picked from had been produced by quenching from high temperature.

A powder diffraction study of the selenide analogue CZTSe [55] revealed the nature of the phase transition as $\mathrm{Cu}-\mathrm{Zn}$ order-disorder phase transition. Synchrotron data were collected at beamline KMC-2 [56, 57] at BESSY II (HZB, Berlin, Germany). Data in this experiment were collected four different energies below the K-absorption edges, in the ranges $8.919-8.974 \mathrm{keV}(<\mathrm{Cu} \mathrm{K})$ and $9.599-9.654 \mathrm{keV}(<\mathrm{Zn} \mathrm{K})$ as well as off edge $(8.048 \mathrm{keV})$, and one in between the two edges $(9.376 \mathrm{keV})$. Data analysis was done by Rietveld refinement of the individual datasets and subsequent analysis of the energy dependency of the site occupation factors [15] in order to locate problems in the data and refinement process.

Joint refinement for direct determination of the site occupancy factors was found to be unstable in this case. This is not a problem of anomalous scattering; it should be obvious from the list in this review paper that this approach normally works very well. Instead the problem is caused by the symmetry of the phase transition. The space group of disordered CZTS is $I \overline{4} 2 m$; ordering within the $\mathrm{CU}-\mathrm{Zn}$ plane reduces this to $I \overline{4}$. The symmetry break is thus merohedric, does not change the Bravais lattice. As a result, all Bragg reflections that become different due to the phase transition are superimposed in powder diffraction. In single crystals, merohedric twinning often results in the same situation. This makes it particularly hard to reliable determine the symmetry-breaking cation order.

In contrast for example, silver indium telluride $[38,39]$ also has a crystal structure derived from the sphalerite type by cation ordering. The disordered form has the same symmetry as disordered CZTS, $I \overline{4} 2 \mathrm{~m}$ with a doubling of the c-lattice parameter compared to the cubic unit cell of sphalerite. The complete ordering of the cations, however, breaks the symmetry in a different way, resulting in space group $P \overline{4} 2 c$. The phase transition breaks the l-centering, giving rise to a large number of Bragg peaks that are extinct in high-symmetry phase. This vastly reduces the problems faced in the structural analysis.

\section{Enhancement of contribution of specific atoms to structure features}

Besides site occupation, varying the scattering power of an element can also be used for the enhancement of its contribution to other structural features. MEAD was applied to $\left(\mathrm{TaSe}_{4}\right)_{21}$ [58]. This material is a quasione-dimensional metal; these compounds exhibit Peierls transitions, where the material changes from a metallic to a semiconducting or isolating state, associated with a modulation of the metallic atoms. This compound exhibits infinite $\left(\mathrm{TaSe}_{4}\right.$ ) chains, for which one would expect a low-temperature Peierls transition and tetramerization of the Ta atoms with corresponding modulation. Instead, an "acoustic" modulation affecting all atoms was found. Resonant diffraction around the Ta L-III edge was used to study specifically the modulation of the Ta atoms. The intensities of various Bragg peaks and their satellites were collected in an energy range covering the Ta L-III edge, using scans $9.830-9.930 \mathrm{keV}$. It was found that some satellite reflections exhibit a contribution from the Ta atoms, thus change intensity at the absorption

edge, whereas other modulation peaks do not. Thus there exists a modulation vector for the Ta atoms 
corresponding to the expected Peierls modulation. By using resonant scattering, this expected modulation of the metallic atoms could be isolated from the dominant acoustic modulation.

\section{Presence of atoms in a given crystal phase}

Like with Rietveld refinement, when analyzing individual diffraction peaks it is in principle sufficient to measure at a single energy close to the absorption edge. This approach was utilized in the analysis of the atomic ordering of FeCo-phase in nanocrystalline Fe-Co-Nb-B alloys $[59,60]$.The analyzed compounds, Fe ${ }_{81-x} \mathrm{Co}_{x} \mathrm{Nb}_{7} \mathrm{~B}_{12}$ with $\mathrm{x}=20-61$, are a soft magnet, metallic glass known as Hitperm alloy. The performance of these compounds depends on the presence of a nanocrystalline ferromagnetic phase. It could be shown that in the partially nanocrystalline state, Co-poor samples are disordered, while in Co-rich sample an ordered $\alpha^{\prime}-$ FeCo phase ( $\mathrm{CsCl}$ type) is present. This ordered superstructure does not reveal itself in normal X-ray diffraction due to the similar atomic scattering factors of Fe and Co. But the 100 super-lattice reflection could be observed in Co rich alloys by choosing the $\mathrm{x}$-ray energy close to the Fe K edge. It should be noted that Fe and Co sport extremely high contrast in neutron diffraction. This is an example of an experiment that should ideally be conducted at a neutron source for best results, provided a sufficient amount of sample is available.

Energy scans over the K-edge of cobalt were used to check the substitution of cobalt for titanium and zinc in $\mathrm{TiO}_{2}$ and $\mathrm{ZnO}_{2}$ thin films, respectively [61]. Co-doped Titanium oxide, $\mathrm{TiO}_{2}$ is an important diluted magnetic oxide semiconductor. One of the important questions regarding the origin of the magnetism was, whether the observed ferromagnetism was caused intrinsically from Co spins randomly substituted on the Ti sites or from segregated Co clusters. X-ray fluorescence was used to confirm the presence of Co atoms in the observed area. The intensity of the 202 Bragg peak was then measured in the energy range $7.6-7.8 \mathrm{keV}$. For $\mathrm{Zn}_{1-\mathrm{x}} \mathrm{Co}_{x} \mathrm{O}_{2}$ the expected decrease in intensity at the absorption edge shows that $\mathrm{Co}$ has been introduced into the $\mathrm{Zn}$ sites of the crystal structure. For $\mathrm{Ti}_{1-\mathrm{x}} \mathrm{CO}_{\mathrm{x}} \mathrm{O}_{2}$, both in is rutile and in its anatase form, no intensity anomaly was observed. As the calculated intensity, assuming that the Co present does substitute Ti, predicted significant intensity changes, this is clearly not the case.

\section{Thin films on substrate}

The analysis of thin films on substrates is a common task in semiconductor analysis, as for many applications (e.g. photovoltaics) this is the form in which the compound might eventually see technical use. This results in multiple complicating factors: The very small sample volume give only a weak signal with low signal-to-noise ratio. Often the signal from the actual thin-film sample is dwarfed by the contribution from the substrate. Strong preferred orientation up to epitaxy can occur and has to be considered in the analysis. Bragg peaks are not well-defined points in reciprocal space, but crystal trunctation rods (CTR); special care during data collection is thus necessary. All this combined is sufficient to explain why analysis of thin-film compound semiconductors was mostly done using the more robust MEAD approach instead of joint Rietveld refinements: 
MEAD has been used to analyze the distribution of $M n$ in epitaxial layers of (Ga,Mn)As, successfully determining the densities of $\mathrm{Mn}$ ions in substitutional and interstitial positions in the GaAs lattice [62, 63]. Data were collected using in the range 6.4 to $6.7 \mathrm{keV}$, around the Mn K-edge. The data are normalized to the corresponding substrate peak, which as pure GaAs is not affected by the edge. This helps in avoiding systematic errors like energy dependence of the beam intensity.

Thin films of ferroelectric bismuth lanthanum nickel titanate with varying B-content [64] have been studied by analyzing the energy dependence of the 001 reflection near the Bi-L III edge. Analysis in this paper was qualitative only; the presence of a cusp of the intensity due to the anomalous dispersion at the Bi $\mathrm{L}$ III-edge energy was taken as proof that $\mathrm{Bi}$ forms a solid solution with the stoichiometric structure. The direction of the intensity change showed that $\mathrm{Bi}$ occupies the $\mathrm{A}$ site of the perovskite structure, as the intensity change for the B-site would be in the opposite direction. Also, the dip in the x-ray intensity decreased systematically with decreasing Bi content as expected for complete uptake of the extra Bi into the structure.

Structural and chemical ordering of $\mathrm{Co}_{x} \mathrm{Mn}_{y} \mathrm{Ge}_{z}$ epitaxial films on $\mathrm{Ge}$ (111) also has been studied by multiple-edge anomalous diffraction [19]. The interest in $\mathrm{Co}_{2} \mathrm{MnGe}$ stems not from semi-conductivity, but from its nature as a half-metallic Heusler alloy [65]. Intensity scans of selected Bragg peaks were done at the K-edges of Co, Mn, and Ge, hence all three elements in the compound, with a scan width of a few hundred $\mathrm{eV}$. Fits were then done to the ratio of two peaks using structure factors as function of site occupancy. This allowed the determination of site-specific chemical disorders, including site swapping, antisites, and vacancies, and their dependence on alloy concentration, revealing high levels of $\mathrm{Mn}-\mathrm{Ge}$ swapping and moderate levels of Ge antisites and Co site vacancies. A similar experiment on the same alloy had been conducted at earlier times by the same group [66], emphasizing the proof-of-concept aspects.

We did only find one published case of "conventional" joint refinement of CTR data sets. The system under analysis in this case was a thin film of $\mathrm{LaAlO}_{3}$ on $\mathrm{SrTiO}_{3}$ substrate [67]. Lanthanum aluminate - strontium titanate interfaces, among other interesting properties, show electrical conductivity far higher than any of the individual components [68]. Vonk did two measurements, one away from the edge at $10.00 \mathrm{keV}$ and around the $\mathrm{La} \mathrm{L}-\mathrm{I}$ edge $(6.280 \mathrm{keV})$ of one unit cell thin $\mathrm{LaAlO}_{3}$ on $\mathrm{SrTiO}_{3}$ substrate. While lanthanum and strontium in principle could be distinguished by normal X-ray diffraction, in this kind of system an unknown, but large, amount of vacancies can be expected. The use of anomalous diffraction allowed in this case distinguishing the various site occupancies unequivocally, demonstrating the presence of both vacancies and $\mathrm{Sr} / \mathrm{La}$ intermixing on sites.

The use of the La L-I edge in this work should be noted in particular. Normally in experiments at the L absorption edge, the L-III edge is selected, as it has the lowest energy. In the case of lanthanum, the energies of the L-edges I, II, and III are 6.266, 5.891, $5.483 \mathrm{keV}$, respectively. With energies this close, the anomalous factors $f^{\prime}$ and $f^{\prime \prime}$ at L-III are still effected by the L-II edge. This limits the contrast that can be achieved by varying $f^{\prime}$ and also will lead to increased background from fluorescence. However, in this energy range already absorption of X-rays by air is significant, an impediment rising very fast as energies get lower. 


\section{Structure determination of thin films}

A particular complication is that the crystal structure of a thin film might be different from the bulk compound with the same composition, making it necessary to determine the structure from scratch. For this a special approach has been developed, applying MAD to but crystal trunctation rod data (called surface truncation rods therein):

The MAD STR algorithm was tested on data from a thin film of $\mathrm{SrTiO}_{3}$ semiconductor grown on $\mathrm{NdGaO}_{3}$ [22]. With very small thickness of the structure under inspection, three monolayers of $\mathrm{SrTiO}_{3}$ in this case, the diffraction pattern does not consist of well-defined Bragg peaks, but of truncation rods. The diffraction patterns were recorded at three energies: below the absorption edge of strontium at $16.0 \mathrm{keV}$, on the absorption edge at $16.11 \mathrm{keV}$ and above the absorption edge at $16.5 \mathrm{keV}$. in contrast to the techniques discussed prior in this review, this approach makes use of the strong change of the imaginary component $f^{\prime \prime}$ of the scattering factor at the absorption edge.

\section{Phase identification by anomalous spectroscopy}

Finally, Diffraction Anomalous Near-Edge Structure spectroscopy (DANES) and its high-quality variant Diffraction Anomalous Fine-Structure Spectroscopy (DAFS) has to be mentioned. Regarding the kind of data that are collected, these techniques are basically the same as the multiple-edge anomalous diffraction (MEAD) used extensively in the analysis of compound semiconductors. The difference lies primarily in the analysis of the data. Where MEAD aims at an analysis through diffraction theory, DANES and DAFS utilize analysis equivalent to XANES and EXAFS, respectively. Diffraction is used only to infuse site-specificity into the results.

Besides the Ga-edge experiments mentioned in passing, DANES has been used for the analysis of titanium and aluminium nitride thin films. The wurtzite phase of aluminum nitride (w-AIN), wurtzite phase (w-AIN) is with a wide band gap a semiconductor material used in deep ultraviolet optoelectronics. Titanium nitride (TiN) shows the electrical properties of a metal, but behaves as a semiconductor when deposited as a thin film [69]. Titanium and aluminium nitride thin films, $\operatorname{Ti}_{1-x} \mathrm{Al}{ }_{x} \mathrm{~N}$, have been analyzed [70] using diffraction anomalous near edge spectra [70]. These films are known to be compromised of crystalline domains with hexagonal, hcp symmetry, but with a high fraction of nanocrystallite matrix and grain boundaries. These can be distinguished, as XANES spectra are averaged on the whole sample, while DANES spectra give only information on the crystals from which the selected Bragg peak originates. For this, DAFS and XAFS experiments were performed at the DIFFABS beamline at SOLEIL, France, in the Ti K-edge region (4900$5600 \mathrm{eV}$ ). The observation of a sharp Ti K pre-edge peak in DANES was then takes as confirmation that Ti atoms are incorporated in the hexagonal lattice. Subsequently, the comparison between DAFS and XAFS reveals that a significant part of $\mathrm{Ti}$ atoms are located in nanocrystallites with cubic symmetry outside of the hcp crystallized columns. 


\section{Experiments on nanostructures}

Beyond atomic-resolution structure analysis, anomalous diffraction has also been applied to the study of nanostructures. The complexity of the techniques applied to this field ranges from quite simple to rather sophisticated.

Free-standing InAs quantum dots on a GaAs substrate have been investigated [71] using grazing incidence $x$-ray diffraction. To suppress the strong scattering contribution from the GaAs substrate, anomalous diffraction at the superstructure reflection was used, showing that the relative intensities from the dots and the substrate undergo a significant change with the $\mathrm{x}$-ray energy below and above the As K-edge. Since the signal from the substrate material can essentially be suppressed, this method is suited for the investigation of strain, shape, and interdiffusion of buried quantum dots and quantum dots embedded in heteroepitaxial multilayers.

Utilizing this effect, growth and microstructure of GaN / AIN quantum dots has been studied extensively at the Ga-K edge using both grazing incidence multiwavelength anomalous diffraction and grazing incidence diffraction anomalous fine structure spectroscopy EDAFS [72-76].

This combined approach using both multiwavelength anomalous diffraction and diffraction anomalous fine structure (DAFS) spectroscopy, in grazing incidence geometry, has also been applied to the study of AIGaN nanowires grown on silicon surfaces [77]. The sample consisted of 1 micron long AIGaN nanowires with the base embedded in a rough $20 \mathrm{~nm}$ thick AlGaN layer. Both the Al content of the wires and the possible presence of an $\mathrm{Al} / \mathrm{Ga}$ concentration gradient had to be determined. Grazing incidence multiwavelength anomalous Diffraction 2D maps were measured at 12 energies across the Ga K-edge, close to the specular GaN(105) and AIN(105) Bragg reflections. As the Ga and the Al+N structure factors of the Al rich spot did not superpose, whereas those of the Ga rich spot did superpose, a composition gradient in the 2D AlGaN layer that grows in between of the wires could be deducted.

Diffraction was then used select different regions of the nanocolumn for grazing incidence diffraction anomalous fine structure spectroscopy analysis (EDAFS), which allowed refinement of the Ga/Al content.

\section{Summary}

Over the years, a wide variety of techniques utilizing anomalous diffraction have been applied to compound semiconductors. However, the total number of experiments done this way is still quite small. Many compound systems have only been analyzed once. The same is true for various techniques and analysis procedures. In other cases, multiple experiments have been conducted, but all by the same group of scientists. This makes identifying trends hard to the degree that there are no clear standard procedures. One trend, however, is quite clear: Whereas in early experiments specialized techniques have been applied, which required a detailed understanding of the physical processes underlying anomalous scattering, in more recent experiments slight variations of standard techniques known from normal diffraction analysis dominate. This is promising as far as future utilization of anomalous scattering by the wider community is concerned. Scientists studying foremost compound semi-conductors, not anomalous 
scattering, face an increasingly lower entry threshold and are thus more likely to judge these experiments worth the time.

\section{References}

1. Schorr, S., The role of point defects in multinary chalcogenide compound semiconductors. Acta Crystallographica Section A, 2014. 70(a1): p. C230.

2. Sears, V.F., Scattering lengths for neutrons, in International tables for crystallography. 2006. p. $444-454$.

3. Lengeler, R., Experimental determination of the dispersion correction $f^{\prime}(E)$ to the atomic scattering factor, in Resonant anomalous X-ray scattering, G. Materlink, C.J. Sparks, and K. KFischer, Editors. 1994, North-Holland: Amsterdam.

4. Cromer, D.T. and D. Liberman, Relativistic Calculation of Anomalous Scattering Factors for XRays. Journal of Chemical Physics, 1970. 53(5): p. 1891-\&.

5. Cromer, D.T. and D.A. Liberman, Anomalous dispersion calculations near to and on the longwavelength side of an absorption edge. Acta Crystallographica A, 1981. 37: p. 267 - 268.

6. Merritt, E.A. X-ray Anomalous Scattering. [cited 2014 June 2014].

7. Henke, B.L., E.M. Gullikson, and J.C. Davis, $X$-Ray Interactions - Photoabsorption, Scattering, Transmission, and Reflection at E=50-30,000 Ev, Z=1-92. Atomic Data and Nuclear Data Tables, 1993. 54(2): p. 181-342.

8. Chantler, C.T., et al., X-Ray Form Factor, Attenuation and Scattering Tables, Version 2.1. 2005, Gaithersburg, MD: National Institute of Standards and Technology.

9. Brennan, S. and P.L. Cowan, $A$ suite of programs for calculating $x$-ray absorption, reflection and diffraction performance for a variety of materials at arbitrary wavelengths. Review of scientific instruments, 1992. 63: p. 850 - 853.

10. Evans, G. and R.F. Pettifer, $\mathrm{CHOOCH:} \mathrm{a} \mathrm{program} \mathrm{for} \mathrm{deriving} \mathrm{anomalous-scattering} \mathrm{factors} \mathrm{from}$ $X$-ray fluorescence spectra. Journal of Applied Crystallography, 2001. 34: p. 82-86.

11. Cromer, D.T., Calculation of Anomalous Scattering Factors at Arbitrary Wavelengths. Journal of Applied Crystallography, 1983. 16(Aug): p. 437-437.

12. Heinke, F., A. Werwein, and O. Oeckler, Cation disorder and vacancies in the sulfosalt-like phase Sn4.11Bi22.60Se38 - A resonant X-ray diffraction study. Journal of Alloys and Compounds, 2017. 701: p. 581-586.

13. Petricek, V., M. Dusek, and L. Palatinus, Crystallographic Computing System JANA2006: General features. Zeitschrift Fur Kristallographie-Crystalline Materials, 2014. 229(5): p. 345-352.

14. Welzmiller, S., et al., Determination of the distribution of elements with similar electron counts: $a$ practical guide for resonant $X$-ray scattering. Journal of Applied Crystallography, 2013. 46: $p$. 769-778.

15. Többens, D.M., et al., Quantitative anomalous powder diffraction analysis of cation disorder in kesterite semiconductors. Powder Diffraction, 2016. 31(3): p. 168-175.

16. Oeckler, O., et al., Atom distribution in SnSb2Te4 by resonant X-ray diffraction. Solid State Sciences, 2011. 13(5): p. 1157-1161.

17. Schneider, M.N., et al., Elemental Distribution and Thermoelectric Properties of Layered Tellurides 39R-M0.067Sb0.667Te0.266 ( $M=G e, S n)$. Chemistry-a European Journal, 2012. 18(4): $\mathrm{p}$. 1209-1218.

18. Hodeau, J.L., et al., Resonant diffraction. Chemical Reviews, 2001. 101(6): p. 1843-1867. 
19. Collins, B.A., et al., Structural and chemical ordering of Heusler CoxMnyGez epitaxial films on Ge (111): Quantitative study using traditional and anomalous $x$-ray diffraction techniques. Physical Review B, 2015. 92(22).

20. Kawaguchi, T., K. Fukuda, and E. Matsubara, Site-and phase-selective $x$-ray absorption spectroscopy based on phase-retrieval calculation. Journal of Physics-Condensed Matter, 2017. 29(11).

21. Hendrickson, W.A., Anomalous diffraction in crystallographic phase evaluation. Quarterly Reviews of Biophysics, 2014. 47(1): p. 49-93.

22. Pauli, S.A., et al., Atomic imaging and direct phase retrieval using anomalous surface $x$-ray diffraction. Journal of Physics-Condensed Matter, 2012. 24(30).

23. Wulf, R., Experimental Distinction of Elements with Similar Atomic-Number Using Anomalous Dispersion (Delta-Synthesis) - an Application of Synchrotron Radiation in Crystal-Structure Analysis. Acta Crystallographica Section A, 1990. 46: p. 681-688.

24. Kasap, S. and P. Capper, Springer Handbook of Electronic and Photonic Materials. Springer handbooks. 2007: Springer US.

25. Ioffe, Electronic archive: New Semiconductor Materials. Characteristics and Properties. 2017, loffe Institute: Russia.

26. Infogalactic. List of semiconductor materials. Available from: https://infogalactic.com/w/index.php?title=List_of_semiconductor_materials\&oldid=720494910

27. Mark, H. and L. Szilard, Ein einfacher Versuch zu Auffindung eines selektiven Effektes bei der Streuung von Röntgenstrahlen. Zeitschrift für Physik, 1925. 33: p. 688 - 691.

28. Nishikawa, S. and K. Matukawa, Hemihedry of Zincblende and X-ray reflexion. Proceedings of the Imperial Academy (Japan), 1928. 4(3): p. 96 -97.

29. Williams, G.H., Limiting Forms of Isometric Holohedrons. Elements of Crystallography for students of chemistry, physics and mineralogy. . 1890, New York.

30. Kortright, J.B. and A.C. Thompson, X-Ray Emission Energies, in X-ray data booklet. 2009, Lawrence Berkeley National Laboratory Berkeley, CA.

31. Coster, D., K.S. Knol, and J.A. Prins, Zeitschrift für Physik, 1930. 63.

32. Caticha-Ellis, S., Anomalous Dispersion of X-rays in Crystallography. International Union of Crystallography Commission on Crystallographic Teaching. Vol. 8. 1981, Wales: University College Cardiff Press.

33. Ohsumi, K., et al., Reinvestigation of Lillianite Structure with Synchrotron Radiation. Acta Crystallographica Section A, 1984. 40: p. C255-C256.

34. Ohta, M., et al., Low lattice thermal conductivity in Pb5Bi6Se14, Pb3Bi2S6, and PbBi2S4: promising thermoelectric materials in the cannizzarite, lillianite, and galenobismuthite homologous series. Journal of Materials Chemistry A, 2014. 2(47): p. 20048-20058.

35. Wendschuh-Josties, M., Determination of cation distributions by anomalous dispersion. Zeitschrift für Kristallographie, 1994. 209: p. 107 - 112.

36. Kupcik, V., et al., The 5 Circle Diffractometer at Hasylab-Recent Developments. Nuclear Instruments \& Methods in Physics Research Section a-Accelerators Spectrometers Detectors and Associated Equipment, 1986. 246(1-3): p. 624-626.

37. Fahrnbauer, F., et al., (GeTe)(n)SbInTe3 ( $n<=3)$-Element distribution and thermal behavior. Journal of Solid State Chemistry, 2013. 208: p. 20-26.

38. Welzmiller, S., et al., Solid Solution Series between CdIn2Te4 and AgInTe2 Investigated by Resonant X-ray Scattering. Zeitschrift Fur Anorganische Und Allgemeine Chemie, 2014. 640(15): p. 3135-3142. 
39. Welzmiller, S., et al., Silver Indium Telluride Semiconductors and Their Solid Solutions with Cadmium Indium Telluride: Structure and Physical Properties. Inorganic Chemistry, 2015. 54(12): p. 5745-5756.

40. Hodeau, J.-L., et al., Nine crystal multi-analyzer stage for high-resolution powder diffraction between 6 and $40 \mathrm{keV}$. SPIE Proceedings, 1998. 3448: p. 353 - 361.

41. Wang, K., et al., Influence of Bi doping upon the phase change characteristics of Ge2Sb2Te5. Journal of Applied Physics, 2004. 96(10): p. 5557-5562.

42. Kojima, R. and N. Yamada, Acceleration of crystallization speed by Sn addition to Ge-Sb-Te phasechange recording material. Japanese Journal of Applied Physics Part 1-Regular Papers Short Notes \& Review Papers, 2001. 40(10): p. 5930-5937.

43. Welzmiller, S., et al., Layered germanium tin antimony tellurides: element distribution, nanostructures and thermoelectric properties. Dalton Transactions, 2014. 43(27): p. 1052910540.

44. Urban, P., et al., Temperature dependent resonant $X$-ray diffraction of single-crystalline Ge2Sb2Te5. Crystengcomm, 2013. 15(24): p. 4823-4829.

45. Barkhouse, D.A.R., et al., Device characteristics of a 10.1\% hydrazine-processed Cu2ZnSn(Se,S)4 solar cell. Progress in Photovoltaics, 2012. 20(1): p. 6-11.

46. Wang, W., et al., Device Characteristics of CZTSSe Thin-Film Solar Cells with $12.6 \%$ Efficiency. Advanced Energy Materials, 2014. 4(7).

47. Schorr, S., H. Hoebler, and M. Tovar, A neutron diffraction study of the stannite-kesterite solid solution series. European Journal of Mineralogy, 2007. 19(1): p. 65-73.

48. Schorr, S., The crystal structure of kesterite type compounds: A neutron and X-ray diffraction study. Solar Energy Materials and Solar Cells, 2011. 95(6): p. 1482-1488.

49. Ritscher, A., et al., Off-stoichiometric CZTS: Neutron scattering investigations on mechanochemically synthesized powders. Journal of Alloys and Compounds, 2016. 689: p. 271277.

50. Ritscher, A., M. Hölzel, and M. Lerch, The order-disorder transition in Cu2ZnSnS4 - a neutron scattering investigation. Journal of Solid State Chemistry, 2016. 238: p. 68 - 73.

51. Nozaki, H., et al., Crystal structure determination of solar cell materials: Cu2ZnSnS4 thin films using X-ray anomalous dispersion. Journal of Alloys and Compounds, 2012. 524(0): p. 22-25.

52. Washio, T., et al., Analysis of lattice site occupancy in kesterite structure of Cu2ZnSnS4 films using synchrotron radiation x-ray diffraction. Journal of Applied Physics, 2011. 110(7): p. 074511-1-074511-4-074511.

53. Bourdais, S., et al., Is the Cu/Zn Disorder the Main Culprit for the Voltage Deficit in Kesterite Solar Cells? Advanced Energy Materials, 2016. 6(12).

54. Lafond, A., et al., X-ray resonant single-crystal diffraction technique, a powerful tool to investigate the kesterite structure of the photovoltaic Cu2ZnSnS4 compound. Acta Crystallographica Section B-Structural Science Crystal Engineering and Materials, 2014. 70: p. 390-394.

55. Többens, D.M., et al., Temperature dependency of $\mathrm{Cu} / \mathrm{Zn}$ ordering in CZTSe kesterites determined by anomalous diffraction. Physica Status Solidi B-Basic Solid State Physics, 2016. 253(10): p. 1890-1897.

56. Energie, H.-Z.B.f.M.u., KMC-2: an X-ray beamline with dedicated di ffraction and XAS endstations at BESSY II. Journal of large-scale research facilities, 2016. 2: p. A49.

57. Erko, A., et al., KMC-2: the new $x$-ray beamline at BESSY II. Synchrotron Radiation Instrumentation, 2000. 521: p. 415-418.

58. Favre-Nicolin, V., et al., Structural evidence for Ta-tetramerization displacements in the chargedensity-wave compound (TaSe4)(2)I from x-ray anomalous diffraction. Physical Review Letters, 2001. 87(1). 
59. Gupta, P., et al., Effect of Co addition on the atomic ordering of FeCo-phase in nanocrystalline Fe81-xCoxNb7B12 alloys ( $x=20.25,27,40.5,54,60.75)$ : An anomalous diffraction and Mossbauer study. Journal of Applied Physics, 2013. 114(8).

60. Gupta, P., et al., Ordering of FeCo Nanocrystalline Phase in FeCoNbB Alloy: an Anomalous Diffraction Study. Solid State Physics, Vol 57, 2013. 1512: p. 362-363.

61. Matsumura, T., et al., X-ray anomalous scattering of diluted magnetic oxide semiconductors: Possible evidence of lattice deformation for high temperature ferromagnetism. Physical Review B, 2007. 76(11).

62. Holy, V., et al., Density of $M n$ interstitials in ( $G a, M n)$ As epitaxial layers determined by anomalous $x$-ray diffraction. Applied Physics Letters, 2010. 97(18).

63. Horak, L., Study of the structure of ferromagnetic semiconductors by $x$-ray scattering methods, in Department of Condensed Matter Physics. 2014, Charles University in Prague: Prague.

64. Kobune, M., et al., Growth of ferroelectric bismuth lanthanum nickel titanate thin films by rf magnetron sputtering. Journal of Applied Physics, 2007. 101(7).

65. Ouardi, S., et al., Electronic and crystallographic structure, hard x-ray photoemission, and mechanical and transport properties of the half-metallic Heusler compound Co2MnGe. Physical Review B, 2011. 84(15).

66. Collins, B.A., et al., Anomalous x-ray diffraction study of disorders in epitaxial films of the Heusler alloy, Co2MnGe. Journal of Vacuum Science \& Technology B, 2007. 25(3): p. 999-1003.

67. Vonk, V., Surface structure refinement including anomalous crystal truncation rods. Journal of Applied Crystallography, 2011. 44: p. 1217-1221.

68. Ohtomo, A. and H.Y. Hwang, A high-mobility electron gas at the LaAlO3/SrTiO3 heterointerface. Nature, 2004. 427(6973): p. 423-426.

69. Münster, A., K. Sagel, and G. Schlamp, Titanium Nitride and Titanium Carbide as Semiconductors. Nature Communications, 1954. 174: p. 1154 - 1155.

70. Tuilier, M.H., et al., Nano-structured titanium and aluminium nitride coatings: Study by grazing incidence $X$-ray diffraction and $X$-ray absorption and anomalous diffraction. Thin Solid Films, 2012. 526: p. 269-273.

71. Schulli, T.U., et al., Anomalous x-ray diffraction on InAs/GaAs quantum dot systems. Applied Physics Letters, 2002. 81(3): p. 448-450.

72. Coraux, J., et al., Mechanism of GaN quantum dots capped with AIN: An AFM, electron microscopy, and x-ray anomalous diffraction study. Physical Review B, 2006. 74(19).

73. Coraux, J., et al., Grazing incidence diffraction anomalous fine structure study of GaN/AIN quantum dots. Nuclear Instruments \& Methods in Physics Research Section B-Beam Interactions with Materials and Atoms, 2006. 246(1): p. 58-63.

74. Coraux, J., et al., Step-by-step capping and strain state of GaN/AIN quantum dots studied by grazing-incidence diffraction anomalous fine structure. Physical Review B, 2006. 73(20).

75. Coraux, J., et al., In situ resonant $x$-ray study of vertical correlation and capping effects during GaN/AIN quantum dot growth. Applied Physics Letters, 2006. 88(15).

76. Coraux, J., et al., In situ and ex situ grazing incidence diffraction anomalous fine structure study of GaN/AIN quantum dots. Physica Status Solidi B-Basic Solid State Physics, 2006. 243(7): p. 15191523.

77. Katcho, N.A., et al., Structural properties of Ge/Si(001) nano-islands and AlGaN nanowires by Diffraction Anomalous Fine Structure and Multiwavelength Anomalous Diffraction. 14th International Conference on X-Ray Absorption Fine Structure (Xafs14), Proceedings, 2009. 190. 